\title{
Velocity-induced collapses of stable neutron stars
}

\author{
J. Novak \\ Département d'Astrophysique Relativiste et de Cosmologie - UMR 8629 du CNRS, Observatoire de Paris, \\ 92195 Meudon Cedex, France
}

Received 22 May 2001 / Accepted 11 July 2001

\begin{abstract}
The collapse of spherical neutron stars is studied in General Relativity. The initial state is a stable neutron star to which an inward radial kinetic energy has been added through some velocity profile. For two different equations of state and two different shapes of velocity profiles, it is found that neutron stars can collapse to black holes for high enough inward velocities, provided that their masses are higher than some minimal value, depending on the equation of state. For a polytropic equation of state of the form $p=K \rho^{\gamma}$, with $\gamma=2$ it is found to be $1.16\left(\frac{K}{0.1}\right)^{0.5} M_{\odot}$, whereas for a more realistic one (described in Pons et al. 2000), it is $0.36 M_{\odot}$. In some cases of collapse forming a black hole, part of the matter composing the initial neutron star can be ejected through a shock, leaving only a fraction of the initial mass to form a black hole. Therefore, black holes of very small masses can be formed and, in particular, the mass scaling relation, as a function of initial velocity, takes the form discovered by Choptuik (1993) for critical collapses.
\end{abstract}

Key words. stars: neutron - black hole physics - hydrodynamics - relativity

\section{Introduction}

In the early eighties Shapiro \& Teukolsky (1980) addressed the question of formation of black holes in astrophysical collapses. Among others, they asked the (theoretical) question of the minimum mass of a black hole formed by the adiabatic collapse of a stellar core and "In particular, can the effective mass-energy potential barrier associated with equilibrium configurations be penetrated by low-mass cores with substantial inward, radial kinetic energy?". In astrophysical scenarios, black holes can also form from accretion-induced collapse of neutron stars, if the neutron star is part of a binary system or during a supernova event, when part of the envelope fails to reach escape velocity and falls back onto the new-born neutron star. Therefore, one can also ask the question: how much of the inward kinetic energy has to be put into a neutron star into make it collapse to a black hole? Can a neutron star always collapse to a black hole, provided that it gets enough kinetic energy?

If one looks at static neutron star models, these are stable against perturbations if their masses are lower than some maximal mass and the density is also lower than the "critical" density corresponding to this maximal mass (they are located on the so-called stable branch). They can collapse to form a black hole when the central density is higher than the critical one (see e.g.

^ e-mail: Jerome.Novak@obspm.fr
Gourgoulhon 1991). The question is then whether stable neutron stars (i.e. with lower density and mass than the critical ones) can be "pushed", with a certain amount of inward kinetic energy, to form a black hole, and what is the minimal mass of the formed black hole and/or progenitor? The problem of the minimal mass of a black hole has been solved, first from a more mathematical point of view, by Choptuik (1993), who discovered the "critical collapse" phenomena in General Relativity (see also Sect. 4.1 for a discussion). Still, the problem of a stellar core/neutron star with inward kinetic energy has never been completely studied; to our knowledge, only a partial study was done by Gourgoulhon (1992). The aim of this paper is to numerically follow the collapse of stable neutron stars, with an inward velocity profile, and determine the initial conditions necessary to obtain a black hole as a result of the collapse; the purpose is also to allow for a realistic equation of state (EOS), to study dependence on the EOS, as well as on the initial velocity profile, giving kinetic energy. Therefore, emphasis will be put on neutron star properties in General Relativity and the question of the physical mechanism giving this kinetic energy will not be addressed.

The complete model in General Relativity is described in Sect. 2, including the system of partial derivative Eqs. (2.1), the two EOS used (2.2), and the procedure for obtaining initial numerical models (2.3). Time evolution is studied in Sect. 3, where two different possible 
evolutions for neutron stars are given (3.1 and 3.2). Numerical results are displayed in Sect. 4: together with a link with the "critical collapse" paradigm (4.1), properties of initial data (4.2) and dependence on the parameters (4.3) are discussed. Finally, Sect. 5 summarizes the results and gives some concluding remarks.

\section{Evolution of spherically symmetric neutron stars}

\subsection{Field and matter equations}

The equations for the evolution of the matter and gravitational fields are derived from the Einstein equations using the simplifying assumption of spherical symmetry. Spacetime is foliated into spacelike hypersurfaces $\Sigma_{t}$ and equation are written in terms of the classical $3+1$ formalism of General Relativity (see e.g. Arnowitt et al. 1962). The real parameter $t$ is called the coordinate time and it can be shown that, making the choice of polar time slicing and radial gauge, in spherical symmetry the metric can be expressed in the form (a generalization of the Schwarzschild metric, see also Gourgoulhon 1991):

$$
\begin{aligned}
& g_{\mu \nu} \mathrm{d} x^{\mu} \mathrm{d} x^{\nu}= \\
& -N^{2}(r, t) \mathrm{d} t^{2}+A^{2}(r, t) \mathrm{d} r^{2}+r^{2}\left(\mathrm{~d} \theta^{2}+\sin ^{2} \theta \mathrm{d} \phi^{2}\right),
\end{aligned}
$$

where $N(r, t)$ is called the lapse function. The two functions $N$ and $A$ will often be replaced by $\nu(r, t)$ and $m(r, t)$, defined as ${ }^{1}$ :

$\nu=\ln (N)$ and $A=\left(1-\frac{2 m}{r}\right)^{-1 / 2}$.

The 4-velocity of the fluid is denoted $v^{\mu}$, the fluid radial coordinate velocity being thus:

$\frac{\mathrm{d} r}{\mathrm{~d} t}=\frac{v^{r}}{v^{0}}$

and the "physical" fluid radial velocity $U$, as measured locally by the hypersurfaces observer is defined by:

$U=\frac{A}{N} \frac{\mathrm{d} r}{\mathrm{~d} t}$.

Following Romero et al. (1996), the stress-energy tensor was taken to be that of a perfect fluid; so hydrodynamical equations in General Relativity $\left(\nabla_{\mu} T_{\nu}^{\mu}=0\right)$ have been written in the form of a system of conservation laws

$\frac{\partial \boldsymbol{u}}{\partial t}+\frac{1}{r^{2}} \frac{\partial}{\partial r}\left[r^{2} \frac{N}{A} \boldsymbol{f}(\boldsymbol{u})\right]=\boldsymbol{s}(\boldsymbol{u})$,

where $\boldsymbol{u}=\{D, \mu, \tau\}$ is the vector of evolved quantities, $\boldsymbol{f}(\boldsymbol{u})$ the vector of fluxes and $\boldsymbol{s}(\boldsymbol{u})$ the vector of sources (for details see Romero et al. 1996). The evolved quantities

\footnotetext{
${ }^{1}$ We use geometrized units in which the speed of light $c$ and Newton's gravitational constant $G$ are equal to unity.
}

are defined from hydrodynamical variables, baryon and total energy densities in the fluid frame $\left(n_{\mathrm{B}}\right.$ and $\left.e\right)$ by:

$$
\begin{aligned}
D & =A \Gamma n_{\mathrm{B}}, \\
\mu & =(E+p) U, \\
\tau & =E-D,
\end{aligned}
$$

where $p$ is the fluid pressure given by the EOS (see 2.2), $\Gamma=\left(1-U^{2}\right)^{-1 / 2}$ is the Lorentz factor of the fluid, and $E=\Gamma^{2}(e+p)-p$.

The gravitational field equations in radial gauge, polar slicing and spherical symmetry reduce to two equations (with no time evolution of the gravitational field):

$$
\begin{aligned}
& \frac{\partial m}{\partial r}=4 \pi r^{2} E, \\
& \frac{\partial \nu}{\partial r}=A^{2}\left(\frac{m}{r^{2}}+4 \pi r\left(p+(E+p) U^{2}\right)\right) .
\end{aligned}
$$

The system therefore consists of three evolution equations for the hydrodynamical variables (Eqs. (4)) and two constraint equations for the gravitational field (Eqs. (6)-(7)). Finally, an equation of state $p\left(n_{\mathrm{B}}, e\right)$ closes the system.

\subsection{Equation of state}

In this work two different EOS for nuclear matter have been used to describe the microscopic properties of neutron stars. Apart from the initial condition models, which were calculated with an equation of state $p\left(n_{\mathrm{B}}\right)$ for cold, catalyzed matter, the equations of state used were of the form $p\left(n_{\mathrm{B}}, e\right)$. The first EOS used is the well-known ideal gas model:

$p=(\gamma-1)\left(e-m_{\mathrm{B}} n_{\mathrm{B}}\right)$

where $m_{\mathrm{B}}=1.66 \times 10^{-27} \mathrm{~kg}$ is the baryon mass and $\gamma$ is the index. We chose $\gamma=2$ which may mimic relatively well the properties of neutron star matter but always keeps a sound speed lower than $c$ (causal EOS). This EOS has been used not only because it is very convenient from a numerical point of view, but also because it allows for the calibration and test of the code. To obtain initial conditions, the following expression has been used

$p\left(n_{\mathrm{B}}\right)=K n_{0} m_{\mathrm{B}}\left(\frac{n_{\mathrm{B}}}{n_{0}}\right)^{\gamma}$

with $K=0.1$ (as in the study of rotating neutron star models by Salgado et al. 1994) and

$n_{0}=0.1 \mathrm{fm}^{-3}$.

To get a more realistic EOS for nuclear matter interactions, we used the EOS described in Pons et al. (2000). It is a relativistic field theory model supplemented by nonlinear scalar self interactions. Nucleons $(n, p)$ interact via the exchange of $\sigma-, \omega-$ and $\rho-$ mesons. The contribution from leptons is given by its non-interacting form, since their interactions give negligible contributions. Moreover, 
the star being at chemical equilibrium with respect to the weak processes, the neutrino chemical potentials are zero. However, as a difference from Pons et al. (2000), kaon interactions are not considered in this work. The EOS was tabulated and, during the time integration, the interpolation has been done using bi-cubic splines. This may not be as precise as the method presented by Swesty (1996) (using bi-quintic interpolation), but thermodynamical consistency is still preserved. At low densities, this EOS has been smoothly joined with a polytrope. As far as initial conditions are concerned, the same model has been used, but the temperature has been set to zero.

\subsection{Initial conditions}

To get initial numerical conditions, we first obtained stable spherical neutron star models and then added a velocity profile. A stable neutron star is defined by the fact that its central density is lower than the critical one $\left(n_{\mathrm{B}}^{\text {crit }}\right)$, defined by:

$$
\left.\frac{\mathrm{d} M_{\mathrm{g}}}{\mathrm{d} n_{\mathrm{B}}^{c}}\right|_{n_{\mathrm{B}}^{c}=n_{\mathrm{B}}^{\text {crit }}}=0 .
$$

$\left(M_{\mathrm{g}}\right.$ being the total gravitational mass of the star and $\left.n_{\mathrm{B}}^{c}=n_{\mathrm{B}}(r=0)\right)$. The stable model is easily computed, integrating the well-known Tolman-OppenheimerVolkoff (TOV) system, which is the static (all $\partial / \partial t$ terms and $U$ are set to zero) limit of momentum evolution equation in system (4), plus the equations for gravitational fields Eqs. (6)-(7). For the polytropic equation of state $(\gamma=2, K=0.1)$, one has $n_{\mathrm{B}}^{\text {crit }}=3.18 n_{0}$ and the maximal stable mass is $M_{\mathrm{g}}=3.16 M_{\odot}$. For the tabulated EOS of Pons et al. (2000) (see previous section), one has $n_{\mathrm{B}}^{\text {crit }}=10.5 n_{0}$ and the maximal stable mass is $M_{\mathrm{g}}=2.08 M_{\odot}$. The rather high value for maximal mass for the $\gamma=2$ polytropic EOS is linked to an also high value chosen for $K$. Thus mass scales between both EOS used in this work were different. However for polytropes of the form (9) with $\gamma=1+1 / n$, the constant $K^{n / 2}$ has units of length in geometrized units. Following Cook et al. (1994), one can use this constant to set the fundamental length scale of the system. Defining dimensionless quantities as in Cook et al. (1994) $\left(\bar{t}=K^{-n / 2} t, \bar{p}=K^{n} p\right.$, $\left.\bar{n}_{\mathrm{B}}=K^{n} n_{\mathrm{B}}, \ldots\right)$, one can see that in our case all masses (even in dynamical evolution) scale as $K^{0.5}$.

Once the density and gravitational fields are computed, the inward velocity profile is added in the r.h.s. of gravitational field Eqs. (6)-(7). The metric coefficients are then relaxed in order to take into account the contribution from the kinetic energy in the total gravitational mass. Therefore, initial conditions are consistent with the gravitational field equations. The considered profiles were of the form

$$
U(r)=\frac{A(r)}{N(r)} V\left(\frac{r}{R_{\text {surface }}}\right)
$$

where $V(x)$ has one of these forms $\left(x=r / R_{\text {surface }}\right)$ :

$$
\begin{aligned}
& V(x)=\frac{V_{\mathrm{amp}}}{2}\left(x^{3}-3 x\right) \\
& V(x)=\frac{27 V_{\mathrm{amp}}}{10 \sqrt{5}}\left(x^{3}-\frac{5 x}{3}\right)
\end{aligned}
$$

( $V_{\text {amp }}$ is the parameter defining the amplitude of the profile).

Both are such that the minimal value in $[0,1]$ is $-V_{\text {amp }}$, but (12) verifies $V^{\prime}(1)=0$ and (13) has a null divergence at the surface. These profiles correspond to what is usually observed in collapses of neutron stars (see e.g. Gourgoulhon 1991) or in the formation of neutron stars (see e.g. Romero et al. 1996). The initial minimal value of the velocity $U(r)$ in the interior of the star $\left(r \in\left[0, R_{\text {surface }}\right]\right)$ is noted $-U_{\text {min }}$. It shall also be called the initial velocity profile amplitude.

\section{Dynamical scenarios}

Numerical time integration has been done using High Resolution Shock-Capturing schemes (HRSC, see Banyuls et al. 1997) for the hydrodynamical system (4). The metric constraint Eqs. (6)-(7) were integrated using standard finite-differences methods. The need for using numerical methods able to handle shocks comes from the fact that strong discontinuities can form (see Sect. 3.2), as was first observed by Gourgoulhon (1992). Unfortunately, this last study has been limited by the use of spectral methods, unable to handle shocks.

\subsection{Direct collapse}

For a stable star close to the maximal mass, but with an inward velocity profile, there may be two final issues. If the velocity is relatively small in amplitude, the star enters as (theoretically) infinite series of oscillations, no viscous nor radiative damping is present. With higher velocity, a collapse to a black hole occurs "normally", almost as for an unstable neutron star configuration (as in e.g. Gourgoulhon 1991). Figure 1 shows energy $(E)$ and velocity profiles at different moments of the collapse of such a neutron star. It corresponds to an initial configuration of $2.74 M_{\odot}$, where the initial velocity profile is of the type (13), with $V_{\mathrm{amp}}=0.4 c$, which gives an initial minimal radial velocity of $-U_{\text {min }}=-0.6 c$. This collapse has been followed up to $t=0.48 \mathrm{~ms}$, when then central value of the lapse became $5.13 \times 10^{-5}$, with a radial velocity at the outer edge of $-0.99 c$. Due to the choice of polar slicing, which avoids the appearance of singularities, the horizon of a black hole cannot be numerically described. However, the final state is a black hole, but from the "frozen star" viewpoint, with a collapse of the lapse. These criteria have been used in Sect. 4 to determine whether the result of the collapse was a black hole or not, namely a central value of the lapse $N(r=0)<10^{-4}$ with an ingoing radial velocity. If both of these conditions are fulfilled, it seems unlikely that the collapse lead to anything other than a black hole. 
Density of energy seen by eulerian observer

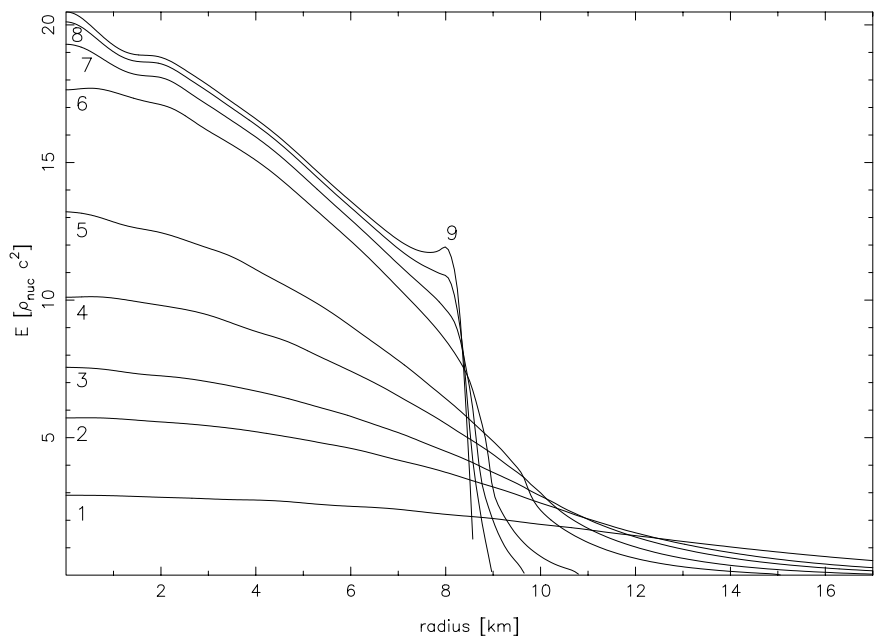

Radial velocity seen by eulerian observer

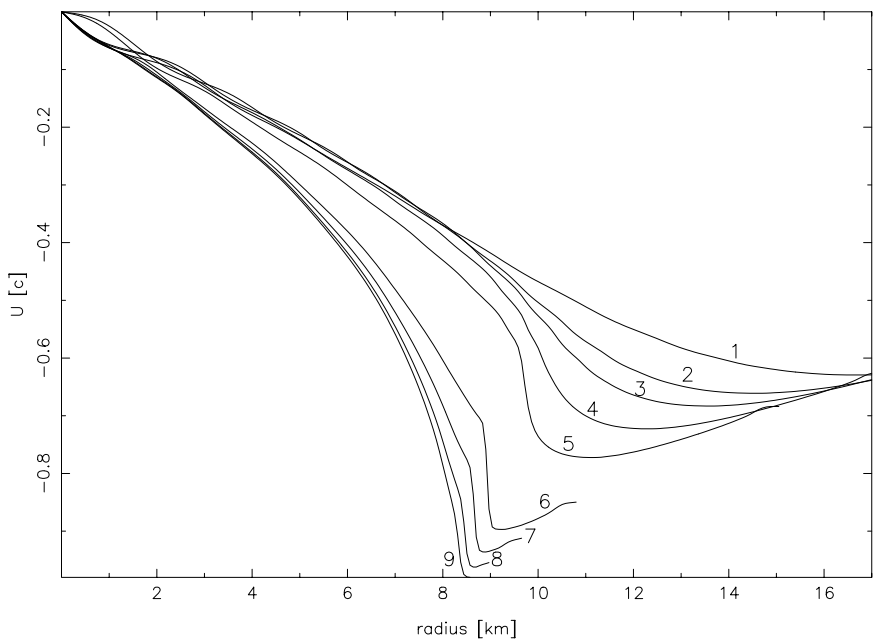

Fig. 1. Snapshots of Eulerian energy density $E$ and radial velocity profiles $U$, for a $2.74 M_{\odot}$ neutron star described by a $\gamma=2$ polytropic EOS, with an initial velocity profile of type (13), and a starting minimal value of the velocity of $-U_{\min }=-0.6 \mathrm{c}$. Labels correspond to the following times: $t_{1}=0.054 \mathrm{~ms}, t_{2}=0.088 \mathrm{~ms}, t_{3}=0.104 \mathrm{~ms}, t_{4}=0.126 \mathrm{~ms}$, $t_{5}=0.149 \mathrm{~ms}, t_{6}=0.202 \mathrm{~ms}, t_{7}=0.225 \mathrm{~ms}, t_{8}=0.248 \mathrm{~ms}$ and $t_{9}=0.271 \mathrm{~ms}$. All profiles have been cut at the surface of the star, defined by the surface density of the initial configuration.

Still, a difference from Gourgoulhon (1991) in the dynamical evolution can be seen in Fig. 1: a strong gradient appears in the velocity profile around $r=9 \mathrm{~km}$, even before the "frozen star" regime (where the metric potentials $A$ and $N$ also exhibit strong gradients). This had already been observed by Gourgoulhon (1992), where it limited the study. It is shown in Sect. 3.2 that, with higher initial velocities, a shock can form and part of the infalling matter can be ejected. In the present "direct collapse" regime all the matter ends in the black hole so that the mass of the resulting object is that of the initial neutron star. In Sect. 4, this has been used to distinguish between both possible types of collapse.

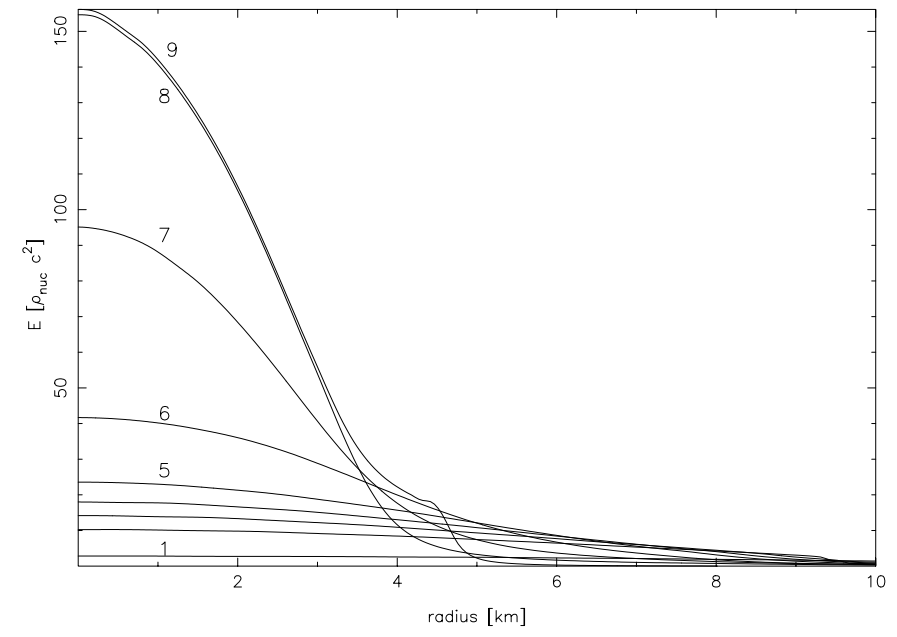

Radial velocity seen by eulerian observer

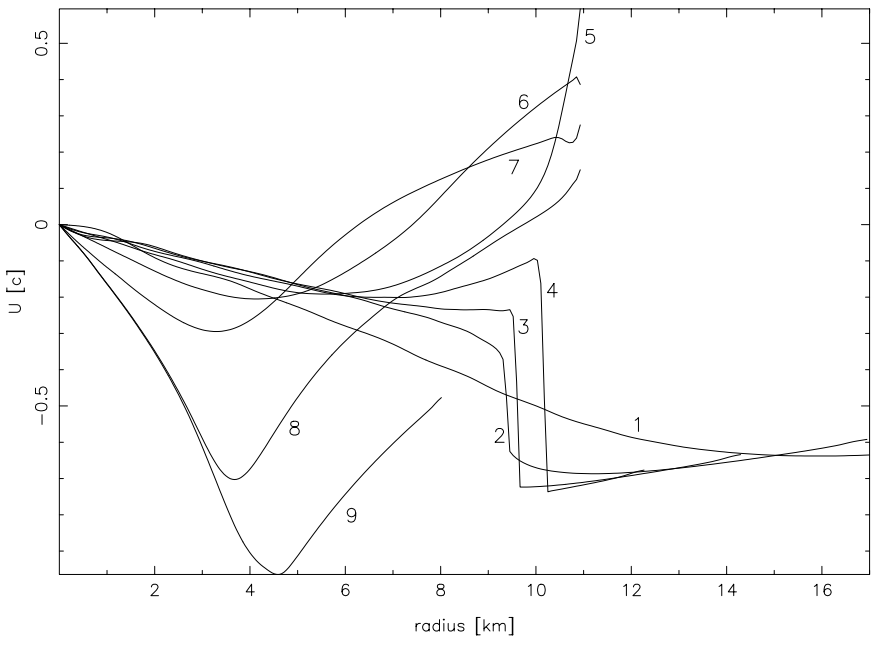

Fig. 2. Eulerian energy density and radial velocity profiles, for a polytropic $\gamma=21.91 M_{\odot}$ neutron star collapse, with an initial velocity profile of type (13), and a starting minimal value of the velocity of $-0.62 c$. The displayed profiles correspond to the following times: $t_{1}=0.064 \mathrm{~ms}, t_{2}=0.123 \mathrm{~ms}, t_{3}=0.143$ $\mathrm{ms}, t_{4}=0.16 \mathrm{~ms}, t_{5}=0.178 \mathrm{~ms}, t_{6}=0.216 \mathrm{~ms}, t_{7}=0.265 \mathrm{~ms}$, $t_{8}=0.348 \mathrm{~ms}$ and $t_{9}=0.477 \mathrm{~ms}$. All profiles have been cut at the surface of the star, defined by the surface density of the initial configuration.

\subsection{Shock and bounce}

For some initial velocities, the "strong gradient" of the previous section turns into a shock, and part of the infalling material is ejected. This can be seen in Fig. 2 where energy density and radial velocity profiles are displayed, for an initial neutron star of $1.91 M_{\odot}$ (the mass is lower than that of Fig. 1 due to a lower initial central baryon density). The shock appears around $t=t_{3}, r=10 \mathrm{~km}$ and then moves out of the numerical grid $\left(t_{4}\right)$ due to the accumulation of matter still falling at $r>10 \mathrm{~km}$. The velocity on the left side of the discontinuity grows and reaches positive values. Later during the collapse, one can see that part of the falling matter starts moving outward and reaches 
escape velocity from the central object $\left(t_{5}\right)$. This of course reduces the possibility of forming a black hole, since matter is spatially less concentrated. Still, the dynamics of the central region (where the velocity is still inward) can proceed to a black hole, which shall then accrete matter which has not reached escape velocity. This has been observed in the collapse displayed Fig. 2 where, at the end of the computation $(t=0.63 \mathrm{~ms})$, the central lapse was $N(r=0)=1.18 \times 10^{-9}$ and the velocity on the edge of the "frozen star" equal to $-0.999 \mathrm{c}$. The central region would collapse "directly", as described before in Sect. 3.1. The final mass of the black hole was $1.61 M_{\odot}$ (and the areal radius in RGPS coordinates equals $4.77 \mathrm{~km}$ ), which shows that almost $16 \%$ of the initial matter of the neutron star has been ejected.

\section{Numerical results}

The dynamical fate of neutron stars with initial velocity are now studied, keeping in mind the different scenarios described in the previous section and varying the mass of the progenitor, as well as the amplitude of the initial velocity profile. This exploration of the parameter space shows the possibility of formation of very low mass black holes, which can be seen as a feature of the "critical collapse" phenomena.

\subsection{Link with critical collapses}

The critical collapse phenomenon (for an interesting review see Gundlach 1999) was discovered in the early $90 \mathrm{~s}$ by Choptuik (1993), who numerically studied the gravitational collapse of spherically symmetric massless scalar field. Depending on some parameter of the initial conditions (which is generically noted $p$ ), the final result of the collapse would be a black hole ( $p$ large) or the dispersion of the field ( $p$ small). He discovered that, when fine-tuning this parameter, he could get black holes of arbitrarily small masses. Moreover, the relation giving the mass of the resulting black hole, close to $p_{*}$, the minimal value to form a black hole, appeared to be universal in the form:

$M_{\mathrm{BH}} \simeq C\left(p-p_{*}\right)^{\alpha}$,

where $C$ is a constant and $\alpha$ is called the critical exponent. The space-time obtained with $p=p_{*}$ shows the very interesting geometrical property of self-similarity.

There have been many works on critical collapses since this pioneering one and, in particular, the study concerning perfect fluids by Neilsen \& Choptuik (2000) is of particular interest for our study. It is shown that perfect fluid collapses, in an ultra-relativistic regime, also exhibit "critical" behavior. Our work can easily be connected to this one, if one considers, at a fixed central density of the initial neutron star, the parameter $p$ to be e.g. the parameter $V_{\mathrm{amp}}$ or the amplitude of the initial velocity profile $U_{\min }$. One can then see that, by finetuning the parameter $V_{\text {amp }}$ ( $U_{\text {min }}$ being a monotonic and continuous function of $V_{\mathrm{amp}}$ ) of (12) or (13), one could

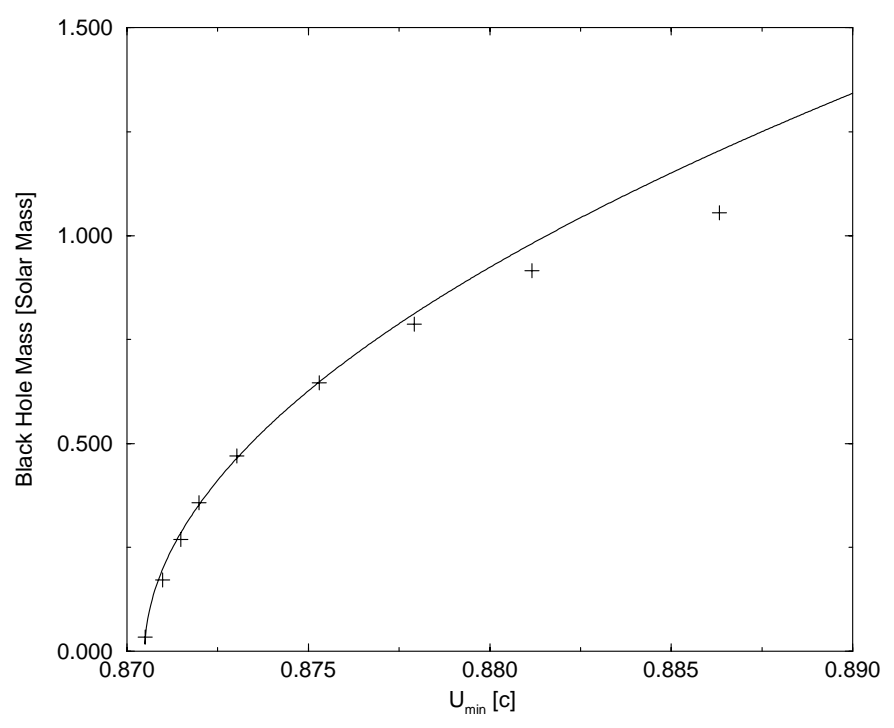

Fig. 3. Masses of the black holes $(+)$ formed through velocityinduced collapses, for a polytropic EOS and with an initial velocity profile of type (13). The initial central density is $0.1 n_{0}$ (see Sect. 2.2) for all the runs. These masses have been fitted by a formula of type (14) (solid line).

get black holes of arbitrarily small masses, as a result of the velocity-induced collapse. We have not investigated the domain of very small black hole masses, for our code was not designed for it. Still, starting with a $1.16 M_{\odot}$ neutron star and an initial velocity profile given by (13) (and $V_{\mathrm{amp}}=0.79732$ ), the result of the evolution was a $3.7 \times 10^{-2} M_{\odot}$ black hole. This corresponds to the lowest black hole mass range (about $10^{-2} M_{\odot}$ ) obtained with our code. In order to test the code against the relation (14), the central density of the initial configuration has been kept constant, and only the parameter $V_{\mathrm{amp}}$ of (13) has been varied (and thus $U_{\min }$ ).

The results displayed in Fig. 3 correspond to the masses of black holes resulting from collapses, if the central density of the initial configurations is held fixed to $0.1 n_{0}$. These masses are drawn (crosses) as a function of the minimal value of the velocity $U$ inside the neutron star. The relation (14) is verified, at least for "small" masses and the best fit (shown on the figure in solid line) corresponds to $\alpha=0.52, C=10.4 M_{\odot}$ and $p_{*}=0.8705 c\left(U_{\min }=p\right)$. These values have been shown to depend on the particular central density used for the initial configurations. The fit is poor for large masses and, in particular, the relation (14) breaks down completely near the maximal mass for stable static neutron stars (when the central density reaches $n_{\mathrm{B}}^{\text {crit }}$ ). The mass scaling exponent $\alpha=0.52$ differs from the result obtained by Neilsen \& Choptuik (2000) $(\alpha=1)$ who used an ultrarelativistic EOS with $\gamma=2$. Although the result from Neilsen \& Choptuik (2000) is rather universal, the study here starts from very different initial conditions (neutron star/exponential distribution of matter in their case). In particular, here, the maximal mass for stable neutron stars gives a mass limit to the problem. Another difference is 
that the parameter used to get the relation (14) is the amplitude of a velocity profile, which has not been studied by Neilsen \& Choptuik (2000). Finally, it has to be stressed out that the family of initial conditions used for this study depends on two parameters (velocity amplitude and central density), while the work of Neilsen \& Choptuik (2000) assumed only a one-parameter family of initial data. The role played by the central density as a parameter is not that of a "critical" one: if one considers the family of initial data given by static neutron stars (without any velocity profile) of increasing central densities, then the value $n_{\mathrm{B}}^{\text {crit }}$ defined by (11) would seem to be the same as $p_{*}$ in controlling the formation of a black hole, but there is no critical behavior at this point. Still, it is interesting to note that the mass scaling relation is valid for velocity-induced neutron star collapses, at least in the limit of low masses (and therefore relatively low central densities).

\subsection{Masses of progenitors - Initial velocities}

Once the EOS and type of velocity profile are chosen, a neutron star model serving as the initial condition for velocity-induced collapse is completely specified by two parameters: the central baryonic density $n_{\mathrm{B}}^{\mathrm{c}}$ and the parameter $V_{\mathrm{amp}}$ or, equivalently, the neutron star gravitational mass and the amplitude of the initial velocity profile $U_{\min }$. For each point $\left(n_{\mathrm{B}}^{\mathrm{c}}, U_{\min }\right)$ in this parameter space the question of knowing whether the corresponding neutron star would collapse to a black hole has been addressed. First, it has been found that there exists a maximal value for $U_{\text {min }}$, for which an initial model of a neutron star could be computed. For higher velocities, it appeared to be numerically impossible to compute the metric coefficients taking into account this large amount of kinetic energy: the relaxation would fail to converge, leading to a velocity $U>c$. This may come from the way these initial conditions are set: the addition of a velocity profile to a static model does not give a well-defined result. Matter is not at equilibrium, while the metric potentials are static. Therefore an improvement of this study would be to consider more realistic initial conditions (as for example in Hawley \& Choptuik 2000), where the velocity profile is not set ad hoc but comes from a dynamical interaction.

This maximal value of $U_{\text {min }}$ depends on the central density of the neutron star, as displayed in Fig. 4; one can see that for central densities going to zero (and therefore also masses), this maximal value goes to one. This figure also shows the various fates of a neutron star in the $\left(n_{\mathrm{B}}^{\mathrm{c}}, U_{\min }\right)$ plane, for the $(\gamma=2)$ polytropic EOS of Sect. 2.2 and an initial velocity profile of type (13). Curves are described from the top-right to bottom-left of the figure. A neutron star set to a point of this parameter space can become a black hole or not. Still, some regions are not really interesting to study. The second curve to be displayed selects neutron stars with a mass lower than maximal, as defined in Sect. 2.3. Studying collapses for neutron stars will higher masses is not relevant since one

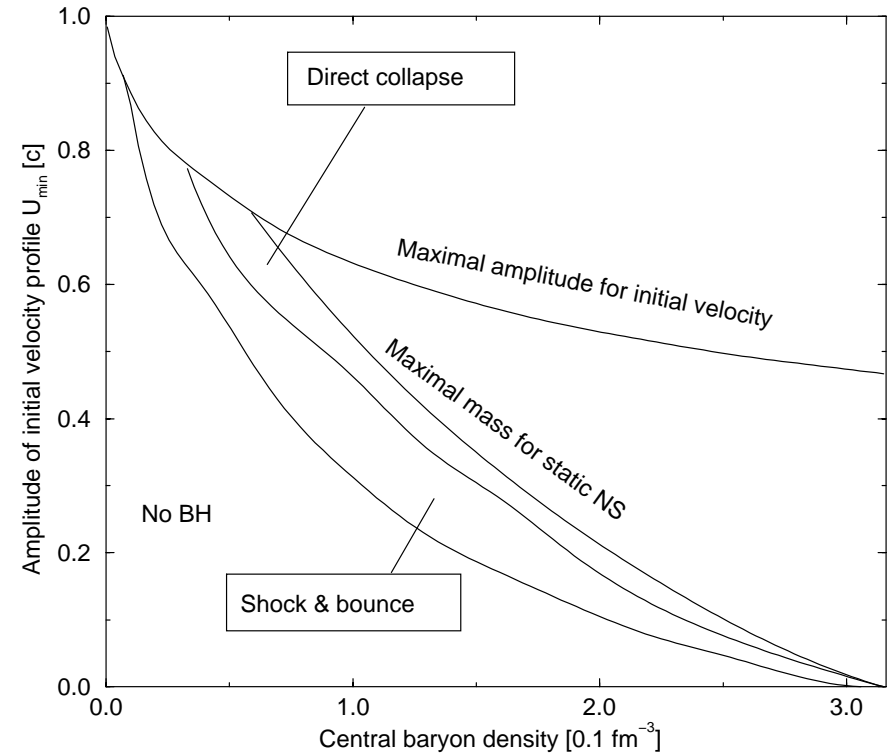

Fig. 4. Parts of the parameter space plane $\left(n_{\mathrm{B}}^{\mathrm{c}}, U_{\min }\right)$ defining the fate of a neutron star with a polytropic $\operatorname{EOS}(\gamma=2)$ and an initial velocity profile defined by (13).

knows that in that case the neutron star will end as a black hole; the additional velocity profile of the neutron star only gives kinetic energy, so that the total mass of the star become larger than the maximal one for stars at rest. It was then observed that this collapse generally proceeds "directly", i.e. no shock is present. One more curve gives the limit between the direct collapse domain and the part of the parameter space for which the star undergoes a shock and bounce, as described in Sect. 3.2. Finally, if the parameters of the initial neutron star model are below the last curve, the black hole does not form, the matter being ejected to infinity. The same regions are displayed in the $\left(M_{\mathrm{g}}, U_{\min }\right)$ plane, in Fig. 5 . In particular, one can see that there exists a minimal mass for the initial neutron star, to form a black hole. This mass is, in the case of a polytrope $\gamma=2$ and an initial velocity profile (13):

$M_{\min }=1.164 M_{\odot}$.

This figure also shows the minimal neutron star mass for which direct collapse (i.e. no ejection of matter) can occur, it is found to be $2.276 M_{\odot}$.

\subsection{Dependence on EOS and velocity profiles}

Although the velocity profile (13) is supposed to occur in most physical scenarios of the gravitational collapse of compact stars, one has to check the dependence of results of previous sections on a change of this velocity profile. Therefore the study of Sect. 4.2 has been undertaken with the initial velocity profile for neutron stars being given by (12). For initial configurations, one can see that this change in the velocity profile induces, for given values of $n_{\mathrm{B}}^{\mathrm{c}}$ and $U_{\min }$, a change in the distribution of kinetic energy in the star and thus a change in the total gravitational mass $M_{\mathrm{g}}$, with respect to the velocity distribution given 


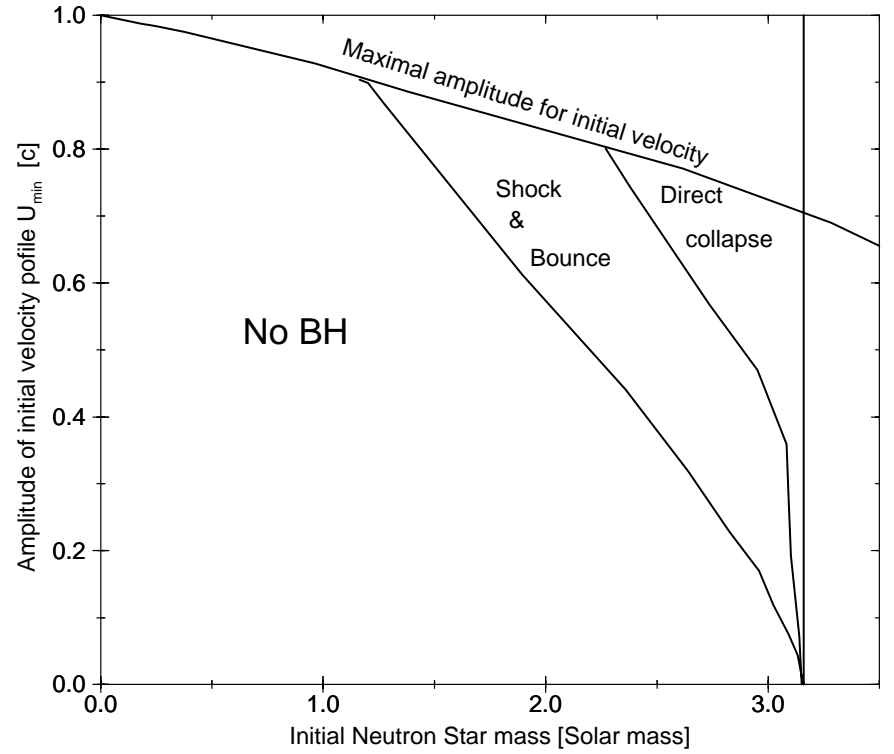

Fig. 5. Parts of the parameter space plane $\left(n_{\mathrm{B}}^{\mathrm{c}}, M_{\mathrm{g}}\right)$ defining the fate of a neutron star with a polytropic EOS $(\gamma=2)$ and an initial velocity profile defined by (13).

by (13). Still, qualitative results concerning collapses were retrieved: direct collapse or bounce as dynamical scenarios and formation of low-mass black holes by fine-tuning of the parameter $U_{\text {min }}$. The scaling relation (14) has been studied for a fixed central density (chosen to be $0.1 n_{0}$ as in Sect. 4.1) and the best fit of results gives the relation:

$M_{\mathrm{BH}} \simeq 6.98 \times\left(U_{\min }-0.8991\right)^{0.52} M_{\odot}$,

for which the exponent $(\alpha=0.52)$ is the same as that obtained using velocity profile (13). The two other coefficients (noted $C$ and $p_{*}$ in Sect. 4.1) are different for the reasons stated above, concerning the difference in kinetic energy distribution. The borders of different regions of Fig. 5 also change slightly, nevertheless we get as a minimal mass for the initial neutron star to form a black hole for a $\gamma=2$ polytrope and an initial velocity profile (12):

$M_{\min }=1.155 M_{\odot}$.

This is less than $1 \%$ different from the minimal mass for velocity profile (13), that is within global numerical error. One then may suppose that this quantity is little dependent on the particular type of velocity profile chosen.

The second global "parameter" one would like to change is the EOS for nuclear matter, since a rather important uncertainty exists on properties of neutron star matter. In particular, the $\gamma=2$ polytrope used in this study had the advantage of rapid computation as well as numerical stability, combined with a rather good description of neutron matter properties. But if one wants to go further, it is then necessary to use a more realistic EOS, as for example the one described in Sect. 2.2. As stated in that section, maximal mass for static neutron stars is found to be $M_{\mathrm{g}}^{\text {crit }}=2.08 M_{\odot}$, for a corresponding central density of $n_{\mathrm{B}}^{c}=10.5 n_{0}$. Therefore, results from computations using this second EOS would show lower masses and higher central densities than those using the analytical EOS. The parameter space $\left(n_{\mathrm{B}}^{\mathrm{c}}, U_{\text {min }}\right)$ has been studied, using the velocity profile given by (13). As for the $\gamma=2$ polytrope, neutron stars with realistic EOS would collapse to form a black hole, provided that the amplitude of the velocity profile is large enough. This collapse could occur directly, as in the case of unstable neutron stars or with the bounce of a part of the matter, which would be ejected, allowing only for the central region to form a black hole. The mass scaling relation is recovered, for relatively small initial central densities; for example, with $n_{\mathrm{B}}^{\mathrm{c}}=1.5 n_{0}$, the following relation is found:

$M_{\mathrm{BH}} \simeq 5.79 \times\left(U_{\min }-0.7519\right)^{0.71} M_{\odot}$,

which has been tested for black holes in the mass range $5 \times 10^{-3}-0.7 M_{\odot}$. The global topology of regions described in Figs. 4 and 5 is kept and the minimal mass for a neutron star (with matter described by this realistic EOS) to form a black hole is found to be

$M_{\min }=0.36 M_{\odot}$.

During the collapses leading to very low-mass black holes (a few $10^{-2} M_{\odot}$ ), the central regions of the star would reach very high densities and it has been checked that sound velocity had never become higher than $c$.

\section{Summary and conclusions}

With an important amount of inward kinetic energy, stable neutron stars may collapse to a black hole, overcoming the potential barrier which separates both types of objects. However, there seem to exist an absolute lower mass limit, depending on the particular equation of state used to describe neutron star matter properties, below which neutron stars cannot pass this barrier. This lowerlimit mass has been found to be $\simeq 1.16 M_{\odot}$ for a $\gamma=2$ polytropic EOS, and $\simeq 0.36 M_{\odot}$ for a more realistic model of nuclear matter. It seems also that these values are independent of the particular initial velocity profile added to static neutron star models. But, as it has been noted in Sect. 2.3, for the polytropic EOS, like all masses this lower-limit mass scales like $K^{0.5}$.

For both studied EOS, in the case when the star collapses to a black hole it may either proceed directly, as if it were an unstable neutron star, all the matter ending in the black hole; or there may appear a shock and bounce, ejecting a part of the matter to infinity, so that only a fraction of the initial neutron star forms a black hole. With such a mechanism, the resulting black hole mass can be arbitrarily small if one fine-tunes the amount of kinetic energy added to the initial neutron star. This result is in accordance with works by Choptuik (1993) on critical collapses, more precisely the mass-scaling relation (14) also applies here, at least for black hole masses not too close to the neutron star critical mass. The mass scaling exponent $\alpha$ found for the polytropic EOS is rather different from that found, with quite different initial conditions, by Neilsen \& Choptuik (2000) for ultrarelativistic $\gamma=2$ EOS. 
Nevertheless, the velocity necessary to achieve the collapse to a black hole of a typical $1.4 M_{\odot}$ neutron star is enormous (see Fig. 5). Even in the case of a smaller $K$, leading to a smaller maximal mass (see Sect. 2.3), this velocity always remains larger than $0.1 c$. It is therefore difficult to imagine any physical process which could inject such an amount of kinetic energy to a neutron star. Tidal (anisotropic) effects when the neutron star is in a binary system with another compact object may result in the destruction of the star (compressed to a "pancake", as ordinary stars passing near a black hole in Marck et al. 1996) or in its oscillations. It has been argued (e.g. by Mathews \& Wilson 2000) that in binary neutron star system, tidal effects could induce such a compression (i.e. increase of central density) such that the stars could collapse into black holes before their merging. This is very difficult to achieve, since in our calculations it happened that during the collapse the central density could reach a value substantially higher than the critical density $n_{\mathrm{B}}^{\text {crit }}$ (in some cases several times $n_{\mathrm{B}}^{\text {crit }}$ ), whereas the star would not end in a black hole, but would rather be dispersed or enter an infinite series of oscillations. Even in the case of the neutron star instability transition in tensor-scalar theory (see Novak 1998), the velocity is never larger than a few percents of $c$, therefore quite far from values displayed in Fig. 5. Finally, let us mention the recent interesting work by Hawley \& Choptuik (2000) who studied the critical collapses of boson stars, where the interaction with a massless real scalar field can result in a significant transfer of energy from the field to the star, allowing for the collapse to a black hole of stable configurations. In the case of neutron stars the interaction with a gravitational wave seems unlikely to transfer enough energy to lead to the collapse to a black hole.
Acknowledgements. I am indebted to José A. Pons for providing with the realistic equation of state. I also would like to thank Eric Gourgoulhon for his help and very fruitful discussions, José M므 Ibáñez who suggested this study and the anonymous referee for his/her comments.

\section{References}

Arnowitt, R., Deser, S., \& Misner, C. W. 1962 , in Gravitation, ed. L. Witten (Wiley, New York)

Banyuls, F., Font, J. A., Ibañez, J. M., Martí, J. M., \& Miralles, J. A. 1997, ApJ, 476, 221

Choptuik, M. W. 1993, Phys. Rev. Lett., 70, 9

Cook, G. B., Shapiro, S. L., \& Teukolsky, S.A. 1994, ApJ, 422, 227

Gourgoulhon, E. 1991, A\&A, 252, 651

Gourgoulhon, E. 1992, Class. Quantum Grav., 9, S117

Gundlach, C. 1999, Living Reviews in Relativity 1999-4, http://www . livingreviews.org/Articles/Volume2/1999 -4gundlach/

Hawley, S. H., \& Choptuik, M. W. 2000, Phys. Rev. D, 62, 104024

Marck, J. A., Lioure, A., \& Bonazzola, S. 1996, A\&A, 306, 666

Mathews, G. J., \& Wilson, J. R. 2000, Phys. Rev. D, 61, 127304

Neilsen, D. W., \& Choptuik, M. W. 2000, Class. Quantum Grav., 17, 761

Novak, J. 1998, Phys. Rev. D, 58, 064019

Pons, J. A., Reddy, S., Ellis, P. J., Prakash, M., \& Lattimer, J. 2000, Phys. Rev. C, 62, 035803

Romero, J., Ibañez, J. M., Martí, J. M., \& Miralles, J. A. 1996, ApJ, 462, 839

Salgado, M., Bonazzola, S., Gourgoulhon, E., \& Haensel, P. 1994, A\&A, 291, 308

Shapiro, S. L., \& Teukolsky, S. A. 1980, ApJ, 235, 199

Swesty, F. D. 1996, J. Comput. Phys., 127, 118 